renders the infection eventually more difficult to eradicate. The following principles may be enunciated :

1. A fresh infection is more easy to eradicate than one of long standing.

2. The widely accepted practice of periodic injections of emetine, or even inadequate doses by the mouth of other emetine compounds, is not recommended, for it has been indicated that, so far from effecting a cure, this method tends to render $E$. histolytica emetine-fast.

3. Cases, however resistant, can eventually be cured, provided the course of anti-amoebic treatment is sufficiently thorough and prolonged.

4. Quinoxyl is a potent non-toxic drug, causing few disagreeable sequelae. In resistant cases of amoebiasis it produces the most permanent results in combination with emetine bismuth iodide. This treatment is usually well tolerated, provided that minutiae of dosage and timing are observed.

\section{Results of Treatment}

TABLE I.-Showing Nature of Anti-amoebic Treatment on 276 ( $51.5 \%$ of the Series) previously Untreated Cases (110 with Active Amoebae and 166 with Cysts)

Emetine bismuth iodide courses of 20 to 26 grains Emetine periodide courses of 28 to 72 grains with tablets of fel

bovinum
Quinoxyl pills ( 4 grains), plus quinoxyl retention enemata $21 \% \%$$\cdots$ Stovarsol 80 grains (cyst-carrier case)

Combined treatments

Emetine bismuth iodide 20 to 26 grains, plus quinoxyl reten-

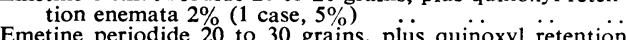
enemata $21 \%$

Auremetine 30 grains, plus quinoxyl retention enemata $2 \frac{1}{2} \%$

29

9
18

198

17

Out of 276 cases there were only $4(1.4 \%)$ uncured. Two definite relapses occurred one year later, and these were ultimately cured with further treatment. In 2 cases the result could not be followed up.

TABLE II.-Showing Effect of Emetine Injection Treatment on 259 (48.5\% of the Series) Cases (138 with Active Amoebae and 121 with Cysts) prior to Parasitic and Clinical Relapse

Emetine injections ranging from 40 to 60 grains, given over a period of 4 to 6 months, in 2 cases reinforced by intravenous emetine

Emetine injections, plus 2 to 3 courses of emetine bismuth iodide,



171 ipecacuanha

TABLE III.-Further Anti-amoebic Treatment of Emetineinjection-treated Cases Detailed in Table II

Emetine bismuth iodide courses of 30 to 40 grains.

Emetine periodide courses of 30 to 70 grains with tablets of $\mathrm{fel}$

bovinum $\ddot{2}$ Quinoxyl pills (4) $\ddot{4}$ grains), plus quinoxyl retention enemata $2 \frac{1}{2} \% \cdots$

Stovarsol 80 grains, plus quinoxyl retention enemata $2 \frac{1}{2} \% \quad \ldots$

Emetol retention enemata

Emetol retention ene

Emetine bismuth iodide 19 to 30 grains, plus quinoxyl retention enemata $21 \%$ (5\% in 16 cases) plus quinoxyl Emetine periodide 30 grains, plus quinoxyl retention enemata $2 \frac{1}{2} \%$. $\ldots$... . . . .

Auremetine $\ddot{30}$ grains, plü quinoxyl retention enemata $2 \frac{1}{2} \ddot{\%}$

RESULTS OBTAINED IN THE SERIES OF TABLE III

Of these 259 there were 20 cases which relapsed still further, but all, with one doubtful exception, were eventually cured. The relapse rate was $7.7 \%$. The following are typical resistant cases:

Case 1.- Infected in Tanganyika in 1917 ; continually relapsed after courses of emetine injections. Admitted to hospital 1920, 1922, 1923, for treatment with E.B.I., E.P.I., and ipecacuanha. Eventually cured in 1928 with full combined course of E.B.I. 30 grains and quinoxyl retention enemata $5 \%$ for ten days.

Case 2.-Infected in Nigeria in 1928 ; had numerous relapses in spite of emetine injections. Had amoebic hepatitis in 1935. Relapsed again after E.B.I. treatment. Eventually cured in 1936 with E.B.I. 20 grains and quinoxyl retention enemata $2 \frac{1}{2} \%$. Reported up to 1941 in good health.

Case 3.-Had spent. forty-two years in West and Central Africa. In 1925 suffered from amoebic dysentery and hepatitis,

controlled by emetine injections. In 1934 E. histolytica cysts were found in the faeces, and sigmoidoscopy revealed amoebic lesions. He was treated by E.B.I. 30 grains and quinoxyl retention enemata $2 \frac{1}{2} \%$. Febrile attacks recurring in 1935, he was operated upon for cholecystitis, but instead a chronic intralobar abscess in posterior part of right lobe of liver was exposed. During convalescence an acute attack of amoebic dysentery developed, with active $E$. histolytica. This was finally cured by repeating the course of treatment with E.B.I. and quinoxyl $5 \%$. He has remained in good health ever since. It is a matter for speculation why the treatment which appeared ineffective in 1934 was curative a year later.

Bibliography

Acton, H. W., and Chopra, R. N. (1933). Ind. med. Gaz., 68, 6.

Birt, E. (1923). Münch. med. Wschr., 70, 205.

Clark, H. C. (1925). Amer. J. trop. Med., 5, 157

Craig, C. F. (1935). Amebiasis and Amebic Dysentery, p. 279, Baillière, London.

Dale, H. H. (1916) Lancet, 2, 183

DuMez, A. G. (1915). Philipp. J. Sci. (B), 10, 73.

Low, G. C., and Dobell, C. (1916). Lancet, 2, 319

Manson-Bahr, P. H., and Morris, R. M. (1925). Ibid., 2, 54

and Sayers, E. G. (1927). British Medical Journal, 2, 490

Marchoux, E. (1923).Bull. Soc. Path. exot., 16, 79.

Martindale, W. H. (1923). Trans. roy. Soc. trop. Med. Hyg., 17, 27.

Mŭhlens, P., and Menk, W. (1921). Münch. med. Wschr., 68, 802.

Reed, A. C., and Anderson, H. H. (1936). Amer. J. med.'Sci., 191, 237.

- David, N. A., and Leake, C. D. (1932). J. Amer. med. Ass., 98, 189.

Rogers, L. (1912). British Medical Journal, 1, 1424.

Vogel, H. (1927). Arch. Schifss- $u$. Tropen-Hyg., 31, 74.

Willmore, J. G. (1923). Trans. roy. Soc. trop. Med. Hyg., 17, 13.

and Martindale, W. H. (1926). British Medical Journal, 1, 525

Yorke, W., Carter, H. F., Mackinnon, D. L., Matthews, J. R., and Smith, A. M. (1917). Ann. trop. Med. Parasit., 11, 87.

\section{AMOEBIASIS-PULMONARY COMPLICATIONS}

BY

\section{B. A. DORMER, M.D., D.P.H., D.T.M. \& H. AND}

\section{J. FRIEDLANDER, M.B., Ch.B., D.P.H.}

(From the King George V Hospital for Tuberculosis, Durban)

Our experience in Natal leads us to believe that pulmonary amoebiasis is fairly common in the coastal belt, more particularly among the non-European population. It is our contention that whenever amoebic dysentery occurs pulmonary complications will be found in a high percentage of untreated cases. This is of great importance to the armies in the East. There is no doubt about the high incidence of amoebiasis in the population of Natal, as judged by the various hospital records. In 1939, for example, at the King Edward VIII Hospital for Natives, Durban, 1,520 cases of amoebic dysentery were admittedi.e., one-tenth of the total admissions for the year. At the McCord Zulu Hospital in 1940, of 3,763 admissions 251 $(6.7 \%)$ were cases of amoebic dysentery. Many of these cases had been wrongly diagnosed as pulmonary tuberculosis.

Amoebiasis of the lung may be primary or secondary. The primary form is comparatively rare; the latter form is much more frequent than is thought.

\section{Primary Pulmonary Amoebiasis}

Manson-Bahr (1940) states that in primary amoebiasis of the lung "the entamoebae reach the lung by direct embolism from the gut wall. Having gained the pulmonary circulation, they form consolidated nodules, which later break down into small abscesses."

Our cases have presented the following signs and symptoms : cough, sputum, haemoptysis, wasting, night sweats, evening rise of temperature-in fact, all those of pulmonary tuberculosis. The main points leading to a diagnosis of primary pulmonary amoebiasis were : (a) a careful history revealing no family or other known contact with a case of 
active open tuberculosis ; $(b)$ a history of diarrhoea or dysentery ; $(c)$ absence of tubercle bacilli in the sputum on repeated examination; $(d)$ the presence of eosinophilia; (e) the presence of cyst forms of Entamoeba histolytica in the stools. On no occasion were amoebae demonstrated in the sputum.

Treatment.-The response of primary amoebiasis of the lung to emetine and carbarsone is remarkable and rapid. Emetine hydrochloride $1 / 2$ grain hypodermically and one carbarsone tablet $(0.25$ gramme $)$ per os are given twice daily at the same time for five days. Then carbarsone alone is given per os twice daily for five days, followed by rest for five days. If the condition has not cleared, the course is repeated.

\section{Secondary Pulmonary Amoebiasis (or Amoebic Abscess of Lung)}

In secondary pulmonary amoebiasis infection of the lung takes place by direct extension from an amoebic abscess of the liver. The abscess may rupture direct into the pleural cavity, giving rise to an empyema. More often the diaphragmatic pleura becomes adherent, thus shutting off the pleural cavity, and extension of the amoebic infection takes place direct into the lung base.

Symptoms and Signs.-The patient complains of cough, and sputum which is blood-stained at times and always characteristically " anchovy-sauce" in appearance, loss of wieight, loss of appetite, malaise, and night sweats. Because of these symptoms the cases are often wrongly diagnosed as basal tuberculosis, more particularly in the Bantu, since it is known that basal tuberculosis is commoner in primitive races than in the European. A careful inquiry into the previous history may elicit a previously unsuspected dysentery. On the other hand, many patients give no history of any bowel disturbances. The cough is often troublesome and the sputum profuse. A mere glance at the characteristic " anchovy-sauce" sputum should direct attention to the correct diagnosis. The absence of tubercle bacilli in the sputum would tend to eliminate pulmonary tuberculosis. Examination of the chest reveals dullness at the right base, with deficient or absent breath sounds and often tenderness in the right hypochondrium, due to the associated hepatitis.

Radiological Appearances.-In primary pulmonary amoebiasis the radiograph may present the appearance of tuberculous infiltration or a bronchopneumonic consolidation. In secondary amoebiasis of the lung there is usually a well-marked opacity at the base, with obliteration of the costo-phrenic angle and immobility of the right hemidiaphragm. The inflammatory reaction spreads from liver to diaphragm, and the process extends then to the lung surface of the diaphragm and to the base of the lung before actual rupture into the lung takes place. The septum between the middle and the lower lobes, usually hardly discernible on the radiograph, takes part in the inflammatory process, hecomes thickened, and appears as a fairly thick band limiting the inflammatory process to the base. On account of the elevation of the diaphragm from the hepatic abscess and the inflammatory products of the base, the septum usually assumes an elevated position with the convexity upwards. When the abscess ruptures into the base the thickened septum often prevents the rupture taking place into the middle lobe. From the base the abscess tends to rupture into the particular lobe bronchus or bronchi, and the patient coughs up the characteristic "anchovysauce" sputum. Often a radiograph will show a small pocket of air lying subjacent to the septum.

Bronchography.-This may be of value in differentiating amoebic abscess from lung abscess due to other causes, since in the latter condition a blocked bronchus is usually found. Basal bronchiectasis is easily distinguished on a bronchogram. We have introduced lipiodol into the right ling base in several cases of amoebic abscess in an effort to show a direct communication between the lung and the liver. As yet we have not been successful in this.

Treatment.-As in primary amoebiasis, emetine and carbarsone have a rapid and dramatic effect. Where an empyema is present, drainage is necessary in addition.

The following are records of seven cases illustrative of pulmonary amoebiasis.

\section{Case I}

This patient, a European aviator aged 32, was employed by Imperial Airways and travelled up and down Africa. About eight months previously he began to complain of cough and blood-stained sputum, which has continued up to the present time. He had lost some weight and his appetite " had gone off." His weight is now stationary. There was no history of any dysentery. He was referred to the King George V Hospital as a case of pulmonary tuberculosis. His personal and family history revealed nothing of note.

On examination his general condition was good. Neither anaemia nor clubbing of fingers was present. Crepitations were heard in the left mid-zone. Nothing abnormal was found in the cardiovascular and genito-urinary systems. The sputum was negative both on direct smear and on concentration. A radiograph showed an area of infiltration in the left lung (Fig. 1).

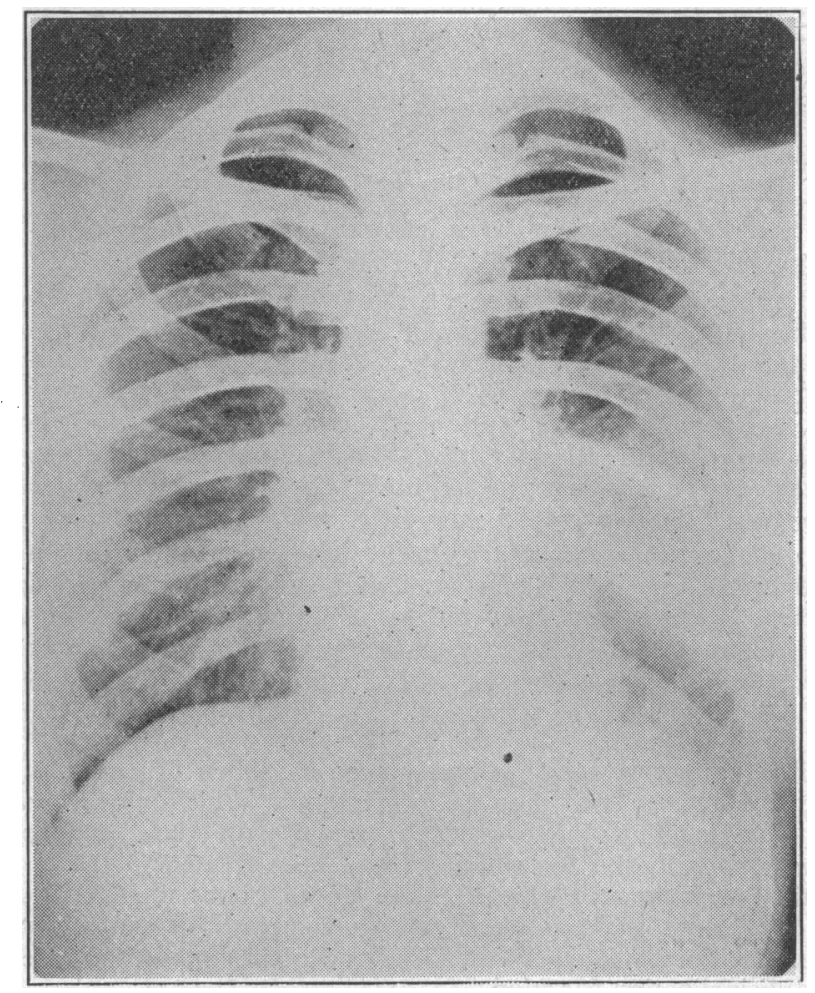

FIG. 1.-Primary pulmonary amoebiasis. Radiograph shows fairly extensive infiltration in left mid-zone and base.

A blood count revealed a $10 \%$ eosinophilia. His stool was examined and cyst forms of $E$. histolytica were found. A course of emetine and carbarsone was given, following which there was rapid and dramatic improvement and his cough and sputum cleared up completely. A radiograph taken after treatment showed the lung fields to be clear (Fig. 2).

\section{Case II}

This patient, a native labourer aged 35 , was seen at a neighbouring hospital, where he was being treated for pulmonary tuberculosis. He gave a history of cough and sputum, bloodstained at times, associated with loss of weight and energy. His 
sputum was repeatedly T.B.-negative. He had also had an attack of "dysentery" some two to three months previously. His general condition was fair ; he had a cough productive of much " anchovy-sauce" sputum, but no clubbing of fingers.

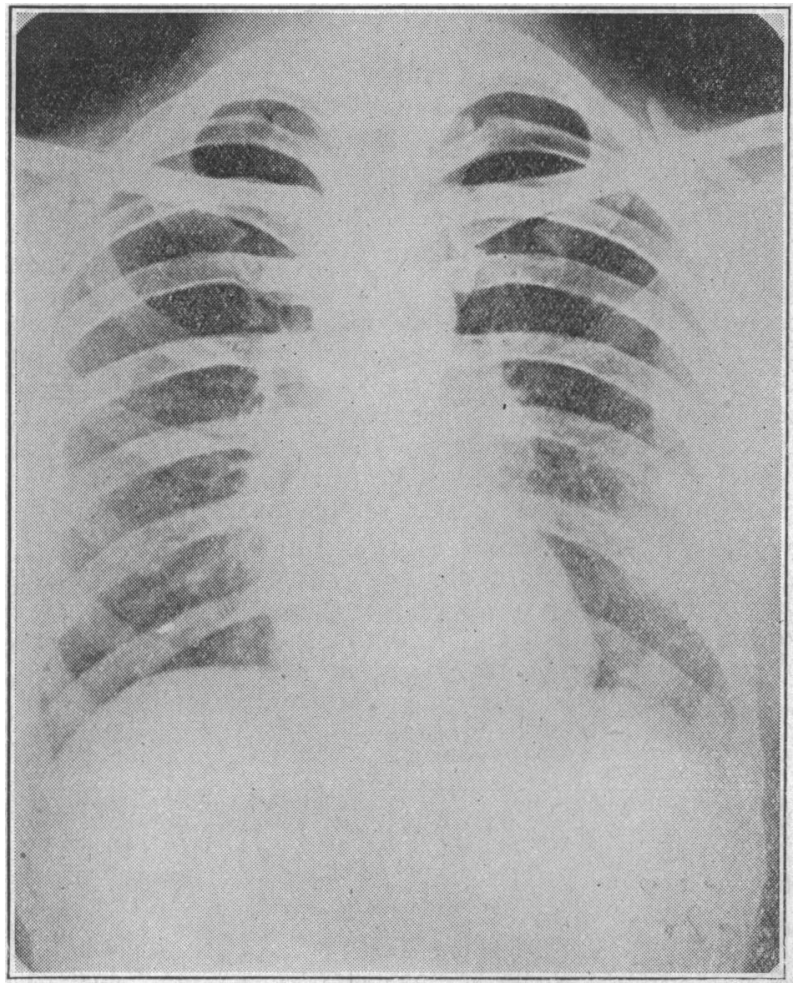

FIG. 2.-Radiograph, taken after one course of emetine, shows an almost complete disappearance of the original lesion.

There was dullness and poor air entry in the lower half of the right lung. The cardiovascular system was normal. The sputum was negative both on direct smear and on concentration. A blood count showed 10,000 white cells per c.mm., with an $8 \%$ eosinophilia. Examination of the stool on three occasions was negative for $E$. histolytica. A radiograph of the chest revealed a fairly massive infiltration at the right base and mid-zone.

A diagnosis of amoebiasis of the lung was made. Emetine and carbarsone were given, with marked improvement in the patient's general condition and great diminution in symptoms. A radiograph taken after two courses of emetine and carbarsone did not reveal any lung lesion.

\section{Case III}

This patient, a native labourer aged 38 , when first seen had been treated for two months for pulmonary tuberculosis. He gave an indefinite history of abdominal pain about eighteen months previously, for which he had been treated in the outpatient department. He felt quite fit till about the last four or five months, when he began to complain of cough and sputum, blood-stained on occasion, and of progressive lassitude and disinclination for food.

On examination his general condition was poor and he looked ill. The temperature was 98 to $100.2^{\circ}$. There was dullness at the right base with poor air entry, and some moist rales were audible. The cardiovascular system was normal. Some tenderness was present in the right hypochondrium. The liver was not palpable. The sputum had an "anchovy-sauce" appearance ; it was profuse and somewhat offensive, and was negative on direct smear and on concentration. Repeated blood counts showed a polymorph leucocytosis up to 12,000 per c.mm., but no eosinophilia. A radiograph revealed a fairly extensive lesion at the right base.

Following treatment with emetine and carbarsone, there was a very rapid and dramatic improvement and his cough and sputum disappeared. The lung lesion was not seen in a radiograph taken after one course of emetine and carbarsone.

\section{Case IV}

A native labourer aged 36 years first developed pain in the right side of his chest six months previous to admission. Cough ensued, with sputum which later became blood-stained. Early in 1939 he had had abdominal pain with frequent blood-stained stools, for which he was "treated" by a native witch-doctor.

On examination he was seen to be very thin. There were cepigmented marks on the face, present from childhood, and slight clubbing of fingers. Breath sounds were absent over the right lower lobe in front, and crepitations were heard posteriorly. The sputum was T.B.-negative. There was tenderness over the liver, the edges of which were palpable.

On admission he was having a frank haemoptysis, but later produced "anchovy-sauce" sputum. A radiograph revealed an opacity at the right base. Amoebic abscess of the lung was diagnosed. No hiatus communicating with the liver detected by bronchography. Following emetine therapy the patieni showed much improvement in general condition, and the lesion at the right base was not present on serial radiographs.

\section{Case $\mathbf{v}$}

This patient, a male native, was a proved case of amoebic abscess of the right base. Bronchography was done in an attempt to outline the hiatus communicating between the liver and the lung. This, however, was unsuccessful. Following bronchography he was treated with emetine and carbarsone, the lesion then clearing completely.

\section{Case VI}

This patient, a native labourer aged 37 , was admitted on May 7, 1940. For the last year or so he had had pain on the right side of his chest and cough associated with periodic attacks of shortness of breath. He described his sputum as "dark yellow" and blood-stained at times. He had had a frank haemoptysis about six months previously. He did not complain of night sweats, and his appetite was good. One sister has a " bad cough." He gave no history of amoebic dysentery, but was treated for roundworms in 1938, and had had an untreated urethral discharge in 1935 .

On examination his general condition was fairly good; he was anaemic and afebrile, and there was clubbing of fingers. Signs of a large cavity were observed at the right base, and there was tenderness over the liver. Sputum was copious but not foul, brownish, and T.B.-negative. A radiograph revealed a cavity at the right base. Bronchography on May 10 showed the lipiodol filling up the bronchi surrounding the cavity, which was well outlined.

A tentative diagnosis of amoebic abscess with cavitation was made, and emetine and carbarsone were given. About three weeks later the cavity had completely disappeared, the cough and sputum had practically ceased, and there was great improvement in the patient's general condition.

\section{Case VII}

This patient, a native labourer aged 22 , had been seen at a neighbouring hospital as a case of pulmonary tuberculosis. $\mathrm{He}$ gave a poor history,' but so far as could be ascertained he began to cough about a month before admission. His sputum had been blood-stained on and off for about two weeks. He complained of progressive loss of weight and energy, loss of appetite, and night sweats. About six months before the coughing began he had had an attack of diarrhoea but could not remember whether he had passed any blood. The diarrhoea disappeared after he had taken one bottle of medicine. About two months earlier he had had some abdominal pain. He was admitted to hospital and a diagnosis of amoebic hepatitis was made, but he left at his own request after receiving only two emetine injections.

On readmission to hospital he looked ill and appeared to be somewhat dyspnoeic. The temperature was 98 to $102^{\circ}$. Clubbing of fingers and pallor of mucous membranes were present. There were dullness and crepitations at the lower half of the right chest, and a few moist rales at the left mid-zone. The cardiovascular system was normal. The liver edge was palpable two fingerbreadths below the costal margin, but was not tender. The sputum was negative on direct smear and on concentration. $A$ radiograph showed infiltration at the right $\cdot$ mid-zone and base 
and some infiltration at the left mid-zone. Cyst forms of $E$. histolytica were found in the stool. There was a leucocytosis of 10,200 per c.mm., with a $7 \%$ eosinophilia.

He was given a course of emetine and carbarsone, and his temperature came down to normal after forty-eight hours. A radiograph taken ten days later showed the lesion on the left side to be cleared, and there was some clearing of the lesion on the right. The course of emetine and carbarsone was repeated, and another radiograph after one month revealed still further clearing of the lesion on the right. At this stage the-white cells numbered 8,700, with a $5 \%$ eosinophilia. Stool examination was negative for E. histolytica.

This patient is feeling much better; his cough and sputum have greatly decreased, and he is anxious to leave hospital. At the time of writing he is still under treatment, but will be discharged as soon as the lesion at the right base has cleared.

\section{Commentary}

The diagnosis of pulmonary amoebiasis is important because of its very rapid response to a specific therapeutic agent-emetine. In the diagnosis various factors must be considered :

(a) History.-A history of blood and mucus in the stools, no matter how long before the lung symptoms are manifest, is an important point. However, many patients do not admit to any previous bowel disturbances.

(b) Site of Lesion.--In secondary pulmonary amoebiasis the lesion is inevitably at the right base.

(c) Stool--Examination of the stool very often reveals the presence of cyst forms of E. histolytica.

(d) Sputum.-The macroscopic appearance of the "anchovy-sauce" sputum is characteristic. Microscopically the sputum reveals an absence of other bacilli, such as tubercle bacilli. In primary amoebiasis $E$. histolytica is said to occur in the sputum, but in our series- of cases, in both the primary and the secondary forms, we have been unable to demonstrate the entamueba.

\section{Differential Diagnosis}

Tuberculosis.-Involvement of the right base with the apices clear is against tuberculosis, except in primitive races, in whom basal tuberculosis is not uncommon. Repeated negative sputum examinations would rule out pulmonary tuberculosis.

Bronchiectasis.-Bronchography would demonstrate the presence of bronchiectatic dilatation at the site of the lesion.

Foreign Body in Bronchus.-Unless there is a clear history of aspiration of a foreign body or the foreign body is easily seen on the radiograph, bronchoscopy is indicated in order to make a diagnosis.

Neoplasms.-A neoplasm may cause bronchial obstruction with resultant atelectasis, which may simulate secondary amoebiasis in the radiograph. Bronchography may show a characteristic " rat-tailing" of the affected bronchus. Bronchoscopy would be of value in differentiating a malignant from an innocent tumour by obtaining a biopsy specimen.

Syphilis of Lung.-As is well known, the symptoms and signs of syphilis are protean in their manifestations. Particularly in the Bantu, syphilis may show itself in the lung as a large tumour or as an extensive infiltration. The presence of a positive Wassermann reaction and the rapid response to the arsenicals would confirm the diagnosis.

Unresolved Pneumonia.-This may show as an opacity on the radiograph. Serial radiographs would show a gradual disappearance of the lesion.

Avitaminosis.-Jersild (1939) reported a case of haemoptysis and lung infiltration caused by avitaminosis. In the
Bantu in South Africa, more particularly in the urbanized native, symptoms of avitaminosis are very frequent. We feel, therefore, that a condition of avitaminosis should be considered in a differential diagnosis.

Where the diagnosis of pulmonary amoebiasis cannot be definitely established we feel that in suspicious cases the patient should be given the benefit of a therapeutic test with emetine and carbarsone.

\section{Summary and Conclusions}

Pulmonary amoebiasis is discussed.

Seven cases of primary and secondary amoebiasis are described.

The importance of examination of the stool, sputum, and blood is emphasized.

Bronchography may be of value in demonstrating a communicating hiatus between the primary lesion in the liver and the secondary lesion at the right base.

All cases of pulmonary amoebiasis show a striking response to emetine and carbarsone.

- We wish to thank Mr. M. E. Gibson, M.S.R., for taking the radiographs.

J ersild, T. (1939). Lancet, 1, 632

Manson-Bahr, P. H. (1940). Manson's Tropical Diseases, 11th ed., London.

\section{THE SURGICAL COMPLICATIONS OF AMOEBIC DYSENTERY}

\author{
BY \\ H. W. S. WRIGHT, M.S., F.R.C.S. \\ Surgeon, E.M.S.
}

The British Empire is at the moment fighting for its existence in a vast area where amoebic dysentery is endemic. So ubiquitous is this disease that about one-fifth of the whole population is involved. After the evacuation of Gallipoli in the last war $23.7 \%$ of 971 cases were infected. In Chiria the percentage of carriers in over 13,000 cases was 20.3 , and amoebic dysentery is the direct cause of between 5 and $10 \%$ of all hospital admissions. Since its effects may extend over a period of from three months to thirty years it is important, at this time, to emphasize that it has significance in every branch of clinical medicine, and will continue to have for many years to come.

It is necessary first to recall some of the pathological features of the primary lesion, which is an ulcerative inflammation of the colon. The Entamoeba histolytica gains access to the large bowel through being able not only to attack and ingest the mucosa but to secrete a ferment which causes coagulation necrosis and tissue lysis. Having reached the submucosa, it multiplies in the centre of a small nutritive cytolysed area, which in its turn, although surrounded by some oedema, is not shut off by an extensive tissue reaction. Spreading in this way, it causes thrombosis of veins and venules, invades the clot, gets into the portal circulation, and is from then onwards caught in the wide mesh of the liver substance. That this is usually a most effective filter is shown by two facts: (1) it was found impossible to produce anything more than cloudy swelling of the liver by injecting very large numbers of amoebae into the portal vein of kittens; (2) amoebae tarely get into the systemic circulation, although amoebic abscess of the brain and the epididymis is well known to occur very occasionally.

It is true that cases of infection of the urinary and even of the uveal tract have been described; but these have not been accepted as genuine by many critical workers. Nevertheless the fact that such records do exist should be a 